\title{
$\beta$-Elemene prevented cisplatin resistance of small cell lung cancer cell H446 by regulating MiR-199a- 5p-mediated autophagy
}

Honglin Li ( $\square$ lihonglin@hb2h.com )

the Second Hospital of Hebei Medical University, Shijiazhuang, China

\section{Research Article}

Keywords: $\beta$-Elemene, MDR, H446, autophagy, MiR-199a-5p, cisplatin, p63

Posted Date: February 9th, 2022

DOI: https://doi.org/10.21203/rs.3.rs-1321845/v1

License: (c) (1) This work is licensed under a Creative Commons Attribution 4.0 International License.

Read Full License 


\section{Abstract}

Background Multidrug resistance (MDR) in small cell lung cancer (SCLC) is a clinical dilemma in chemotherapy. A natural compound, $\beta$-elemene has been found to inhibit MDR cancers. We hypothesized that $\beta$-elemene regulated cisplatin-resistant SCLC by targeting autophagy-related miR-199a-5p.

Methods We assessed the $\mathrm{IC}_{50}$ of multiple chemotherapeutic agents toward SCLC cells H446 and MDR SCLC cells H446/EP with or without $\beta$-elemene using CCK-8. We overexpressed (OE) and knocked down (KD) miR-199a-5p in H446 and H446/EP and determined the expression of miR-199a-5p, autophagyrelated proteins, and the formation of autophagolysosomes using QPCR, western blotting, and MDC staining respectively. We validate the binding of miR-199a-5p to p62 using the Dual-Luciferase assay.

Results $\beta$-Elemene decreased drug resistance of H446 to cisplatin, etoposide, paclitexal, epirubicin, irinotecan, and vinorelbine. Cisplatin increased LC3II/LC3I, ATG5, p62, and the formation of autophagolysosomes in $\mathrm{H} 446$ cells, but not in H446/EP cells, and these effects were blocked by $\beta$ elemene. The OE of miR-199a-5p reduced cisplatin-induced increase of LC3II/LC3I, ATG5, p62, and the formation of autophagolysosomes while the KD of miR-199a-5p did the opposite. the OE of miR-199a-5p reduced the luciferase signal of wild-type p62, but not that of $p 62$ with mutations at the predicted binding site. The effect of $\beta$-elemene on cisplatin-induced autophagy was validated in OE-H446 and KD-H446/EP.

Conclusions $\beta$-Elemene prevented MDR in $\mathrm{H} 446$ and it prevents cisplatin resistance of $\mathrm{H} 446$ by targeting the autophagy regulation of miR-199a-5p.

\section{Background}

More than $80 \%$ of clinical lung cancer cases are diagnosed as non-small cell lung cancers (NSCLC) and only less than $20 \%$ as small cell lung cancer (SCLC) [1, 2], but NSCLC typically grow at a slower rate than SCLC and are difficult to be discovered until they have advanced [3]. As SCLC can be diagnosed at earlier stages, chemotherapy is the major treatment for SCLC instead of surgical treatment, resulting in more drug resistance issues [4]. Multidrug resistance (MDR) is an innate and/or acquired ability of cancer cells to survive against a wide range of chemotherapy drugs [5]. In clinical cancer chemotherapy, MDR has been one of the tough dilemmas. Cancer cells with MDR can expel the anti-cancer molecules outside the cells thereby reducing the absorption of the drug. Although research has revealed many potential mechanisms underlying MDR, the understanding of MDR is still far from complete and no effective way has been found to solve the problem of MDR ideally [6]. Autophagy has been suggested to be one of the factors that might affect MDR [7-9]. It is characterized by a self-digestion pathway that activates lysosomes to degrade damaged or superfluous cell components in the cells $[10,11]$. Studies have shown that autophagy prevents cells from apoptosis, hypoxia, and damage stress responses. As a complex cell behavior, autophagy involved many biological processes and might interfere with MDR pathways [12, 13]. 
In recent years, many naturally occurring compounds have been studied and implemented in the clinical therapy of human disease [14-18]. Accumulating evidence suggests that chemotherapy supplied by traditional medicine might achieve desirable outcomes in clinical cancer treatment, including higher efficiency and lower side effects [19] [20]. A natural compound extracted from Rhizoma zedoariae, $\beta$ elemene has been used for clinical cancer treatment [21] and has shown anti-drug resistance potential in cancer. $\beta$-Elemene has been shown to induce autophagy in human breast cancer cells [22], but whether it affects autophagy in lung cancer has not been studied. A previous study showed that $\beta$-elemene can target lung cancer cells and promote the polarization of macrophages from $M 2$ to $M 1$ to decrease inflammation [23]. In gastric cancer cells, $\beta$-elemene can suppress the metastasis of multidrug-resistant cell lines [24]. In addition, $\beta$-elemene was also found to up-regulate P53 protein expression to facilitate the release of exosomes in lung cancer cells [25]. This inferred that $\beta$-elemene might regulate the membrane system in lung cancer cells, which, we suggested might involve the lysosome membrane in autophagy. Therefore, we believe that $\beta$-elemene might regulated multidrug-resistant lung cancer cells by targeting lysosome-mediated autophagy.

A microRNA (miRNA) miR-199a-5p has been found closely related to autophagy and drug resistance [26]. This miRNA plays roles in multiple cancers, including lung cancer [27-29], laryngeal cancer [30], colorectal cancer [31], etc. A previous study has found that miR-199a-5p inhibited protective autophagy and reversed chemoresistance by regulating DRAM1 protein in leukemia cells [32,33]. Our preliminary bioinformatics study revealed that miR-199a-5p was one of the miRNAs associated with $\beta$-elemene actions (data not shown in this paper). Therefore, we proposed that miR-199a-5p might mediate the effect of $\beta$-elemene and cisplatin on the autophagy of lung cancer cells. In the present study, we explored the correlation between autophagy and MDR development in small cell lung cancer cell (SCLC) H446 cells and investigated the role of miR-199a-5p in this process. Our data supported the use of $\beta$-elemene in clinical chemotherapy for MDR and revealed the role of miR-199a-5p in the autophagy regulation of MDR in SCLC.

\section{Methods}

\subsection{Cell lines and cell culture}

Small cell lung cancer cell (SCLC) line NCl-H446 [H446] (ATCC ${ }^{\circledR}$ HTB-171 ${ }^{\mathrm{TM}}$ ) and lung microvascular endothelium HULEC-5a (ATCC ${ }^{8}$ CRL-3244 ${ }^{\text {TM }}$ ) were purchased from ATCC (Washington, USA). The cell lines were cultured using the RPMI-1640 medium (Gibco; Thermo Fisher Scientific, Inc., Waltham, MA, USA) with $10 \%$ FBS (Gibco; Thermo Fisher Scientific, Inc., Waltham, MA, USA) in a $37^{\circ} \mathrm{C} 5 \% \mathrm{CO}_{2}$ incubator. The multidrug resistance $\mathrm{H} 446$ sub-cell line H446/EP was developed from $\mathrm{H} 446$ with increasing concentration selection of etoposide (Sigma-Aldrich, St. Louis, MO, USA) combined with cisplatin (SigmaAldrich, St. Louis, MO, USA), both increased from $50 \mathrm{ng} / \mathrm{ml}$ to a final dose of 1,000 ng/ml. The H446/EP obtained were cultured in the drug-free medium for over 10 generations before the experiments [34].

\subsection{Cell viability assay}


Cell viability was determined using the Cell Counting Kit-8 (CCK-8, Sigma-Aldrich, St. Louis, MO, USA) assay as a previous study [35]. Briefly, cells were cultured in 96-well plates with drugs accordingly. At the endpoint of the exposure, the CCK-8 reagent ( $10 \mathrm{ml} /$ well) was added. After $3 \mathrm{~h}$ of incubation at $37^{\circ} \mathrm{C}$, the absorbance at $450 \mathrm{~nm}$ was evaluated using a microplate reader (Bio-Rad, Model 680). The cisplatin, etoposide, paclitexal, epirubicin, irinotecan, and vinorelbine were purchased from Sigma-Aldrich (St. Louis, MO, USA).

\subsection{Cell transfection}

The overexpression (OE) and knockdown (KD) of miR-199a-5p were achieved by transfection of sh-miR199a-5p vector or miR-199a-5p expression vector into cells respectively. Briefly, the miR-199a-5p or its shRNA coding sequence was cloned into the pLV-eGFP vectors. Negative expression control vectors (OENC) and shRNA control vectors (KDNC) were also constructed with the same vector. Lipofectamine ${ }^{\circledR}$ 2000 was used to transfect the cells. The transfection was validated by observing the GFP marker in the transfected cells. The vectors were purchased and constructed by Beyotime Biotechnology (Shanghai, China).

\subsection{Western blotting assay}

The protein expressions were analyzed using the western blotting assay as described previously [36]. Briefly, cells were lysed in the RIPA buffer (Sigma-Aldrich, St. Louis, MO, USA) plus protease inhibitor (Pierce Protease Inhibitor Mini Tablets, Thermo Fisher Scientific, Inc., Waltham, MA, USA). SDS-

polyacrylamide gel electrophoresis (PAGE, Sigma-Aldrich, St. Louis, MO, USA) electrophoresis was used to separate the proteins in samples. Then the proteins were transferred to $0.2-\mu \mathrm{m}$ polyvinylidene difluoride membranes (Thermo Fisher Scientific, Inc., Waltham, MA, USA). The membranes were blocked with blocking buffer (Pierce ${ }^{\text {TM }}$ Protein-Free Blocking Buffer, Thermo Fisher Scientific, Inc., Waltham, MA, USA) for $1 \mathrm{~h}$. The membranes were then incubated with primary antibodies at $4^{\circ} \mathrm{C}$, overnight, and then secondary antibodies at room temperature for $2 \mathrm{~h}$ (dilution following the recommended concentration of the antibody respectively). The ECL Detection Reagent (Sigma-Aldrich, St. Louis, MO, USA) was used to visualize the target protein. The primary antibodies used in this study were as follows: LC3B (1:800, 2775S, Cell Signaling); P62/SQSTM1 (1:2500, 18420-1-AP, Protein Tech); ATG5 (1:1000, GTX113309, GeneTex), and $\beta$-Actin (1:5000, sc-1615) (Santa Cruz Biotechnology, Dallas, TX, USA). All the secondary antibodies were purchased from the Abcam (Cambridge, UK).

\subsection{Autophagolysosomes observation}

Monodansylcadaverine (MDC, Sigma-Aldrich, St. Louis, MO, USA) staining was used to observe autophagolysosomes as described previously [37]. Briefly, cells were cultured in a 6-well plate under testing conditions. At the endpoint of the exposure, the cells were incubated with MDC ( $50 \mu \mathrm{mol} / \mathrm{L})$ for 30 min at $37^{\circ} \mathrm{C}$. Then the cells were washed with precooling phosphate-buffered saline (Sigma-Aldrich, St. Louis, MO, USA), followed by the observation using a fluorescence microscope (GXM UltraDIGI-SBMF1, USA). 


\subsection{Real-time quantitative PCR.}

The expression of miR-199a-5p was evaluated using real-time quantitative PCR (QPCR) as described previously [38]. Briefly, TRIzol reagent (Vazyme) was used to extract total RNA from cells following the manufacturer's instructions. The target RNAs were reverse-transcribed to cDNA using the M-MLV-Reverse Transcriptase Kit (Sigma-Aldrich, St. Louis, MO, USA). Real-time PCR analysis was performed using KiCqStart ${ }^{\circledR}$ SYBR $\circledast$ Green qPCR ReadyMix ${ }^{\text {TM }}$ (Sigma-Aldrich, St. Louis, MO, USA) with a Real-Time PCR platform (CFX96, BIO-RAD). All the PCR primers used in the study were synthesized by Thermo Fisher Scientific, Inc. (Waltham, MA, USA). The expression was normalized to RNU6-1 miRNA expression using the $\triangle \triangle C T$ method. The PCR primers sequences were as follows. miR-199a-5p F: 5'ACACTCCAGCTGGGTGTCAGTTTGTCAAAT-3', R: 5'-TGGTGTCGTGGAGTCG-3'; RNU6-1 F: 5'CTCGCTTCGGCAGCACA-3', R: 5'-AACGCTTCACGAATTTGCGT-3'.

\subsection{Dual-Luciferase Reporter Assay.}

Firefly/Renilla Dual Luciferase Assay (Sigma-Aldrich, St. Louis, MO, USA) was used to test the binding of miR-199a-5p to wild-type (WT) or mutated coding sequence of p62 as previously described [39]. Briefly, cells were plated in a 96-well plate and negative plasmids or reporter plasmids with WT or mutated p62 sequence were transfected to cells. The sequences were shown in Fig. $3 \mathrm{G} 2$. After $48 \mathrm{~h}$ transfection, cells were lysed, and the luciferase signal was measured following the protocol with the microplate reader (GXM UltraDIGI-SBMF1, USA).

\subsection{Experiment repetition and statistical analysis}

The CCK assay was repeated three times with 8 biological duplications. The other experiments were repeated three times with three biological duplications. Data are presented as means \pm SD. Student's ttest or one-way ANOVA analysis was used to analyzed significance. A P-value of 0.01 or lower was considered significant.

\section{Results}

\section{1 $\beta$-Elemene concentration screening}

Concentration screening by toxicity is usually the first stop of a pharmacological study [40]. In this study, we first assessed the toxicity of $\beta$-elemene toward SCLC cells H446, drug-resistant H446/EP, and normal lung cells HULEC-5a. Results showed that $\beta$-elemene at $5 \mu \mathrm{g} / \mathrm{ml}$ or lower showed no significant inhibition to cell viability of all three cell lines after $24 \mathrm{~h}$ of exposure. $\beta$-Elemene at $10-100 \mu \mathrm{g} / \mathrm{ml}$ dose-dependently inhibited cell viability of all three cell lines with similar sensitivity of H446 and HULEC-5a, and relatively less sensitivity of H446/EP (Fig.1A). We suggested that the MDR ability of H446/EP accounted for this difference. $\beta$-Elemene at $5 \mu \mathrm{g} / \mathrm{ml}$ or lower were not toxic to cells but according to our preliminary experiments, $\beta$-Elemene at $5 \mu \mathrm{g} / \mathrm{ml}$ was sufficient to affect the action of cisplatin (data not shown in this paper), therefore, we used $5 \mu \mathrm{g} / \mathrm{ml}$ of $\beta$-Elemene in the subsequent study. 


\section{2 $\beta$-Elemene decreased the resistance of multiple chemotherapeutic agents in H446/EP}

Then we did a series of cell viability assays to evaluate the $\mathrm{IC}_{50}$ of multiple chemotherapeutic agents in $\mathrm{H} 446$ and H446/EP cells with or without $\beta$-elemene. This work aimed to calculate the drug resistance index for cancer (DRIC) and investigate the effect of $\beta$-elemene on MDR. Results showed that $\beta$-elemene decreased drug resistance of cisplatin, etoposide, paclitexal, epirubicin, irinotecan, and vinorelbine. The most remarkable DRIC decrease was from cisplatin whose DRIC decreased from 29.95 to 5.56. This decrease in fold change was followed by paclitexal, epirubicin, and irinotecan whose resistance indexes with $\beta$-elemene decreased to lower than one-third of the resistance index without $\beta$-elemene, from 5.57 to 1.48 , from 9.80 to 3.06 , and from 5.84 to 1.61 respectively. The resistance index of etoposide and vinorelbine with $\beta$-elemene decreased to about half of that without $\beta$-elemene, from 58.45 to 27.9 and from 2.09 to 1.05 respectively. (Table 1) These results indicated that $\beta$-elemene prevented $\mathrm{H} 446$ cells from the drug resistance of multiple chemotherapeutic agents. As cisplatin showed the most fold change in drug resistance index, we focused on cisplatin resistance in the subsequent study to further explored the effect of $\beta$-elemene on the drug resistance of $\mathrm{H} 446$ cells. In this study, cisplatin at $5 \mu \mathrm{g} / \mathrm{ml}$ inhibited cell viability of $\mathrm{H} 446$ and H446/EP with $\beta$-elemene but not H446/EP without $\beta$-elemene, thus it is a proper model for us to study the actions of $\beta$-elemene on cisplatin effects.

\section{3 $\beta$-Elemene promoted cisplatin-induced autophagy in H446/EP}

To investigate the relation between autophagy and $\beta$-elemene actions, we determined three indicators for autophagy including the ratio of LC3II and LC3I expression, the level of ATG5, and the levels of p62. Results showed that cisplatin increased LC3II/LC3I, ATG5, and p62 in H446 cells, but not in H446/EP cells. This indicated that the drug resistance of cisplatin was resulted (at least partly) from the insensitivity of autophagy induction. In addition, $\beta$-elemene alone did not make any difference in these indicators, but when it was applied with cisplatin, it increased LC3II/LC3I, ATG5, and p62 in H446/EP cells (Fig.1B-E). This indicated that the combination of cisplatin and $\beta$-elemene significantly increased the autophagy of multi-drug resistance sub-cell line H466/EP compared to the use of cisplatin or $\beta$-elemene alone. To observe the cell activity of autophagy in the cells, we stained the autophagolysosomes with MDC. We found that $\beta$-elemene alone failed to induce the formation of autophagolysosomes while cisplatin only induced the formation of autophagolysosomes in $\mathrm{H} 446$ but not in H446/EP. However, this insensitivity was eliminated by adding $\beta$-elemene to the cells: the combined use of cisplatin and $\beta$ elemene significantly induced the formation of autophagolysosomes in H466/EP (Fig.1F). This further confirmed that $\beta$-elemene promoted cisplatin-induced autophagy in H446/EP.

\subsection{MiR-199a-5p was upregulated in H446/EP}

As mentioned above, miR-199a-5p was involved in MDR. To test whether it was also associated with the drug resistance of H446, we compared the expression level of it in H446 and H446/EP. Results showed that the drug resistance selection increased the level of miR-199a-5p in H446 up to 1,000 times (Fig.2A). Such a remarkable increase in miR-199a-5p expression in H446/EP suggested that miR-199a-5p might 
play a potential role in the drug resistance of H446. To explore the role of miR-199a-5p, we knocked down (KD) and overexpressed (OE) miR-199a-5p in H446 and H446/EP. In H466, the knockdown slightly decreased miR-199a-5p but the overexpression increased miR-199a-5p up to 1,500 folds (Fig.2B). In $\mathrm{H} 466$, the knockdown decreased miR-199a-5p by more than $60 \%$ and the overexpression increased the miR-199a-5p level by 150\% (Fig.2C). If we normalized all data to the endogenous level of miR-199a-5p in $\mathrm{H} 446$, the overexpression increased about 1,500-fold of H446 endogenous miR-199a-5p in both H446 (from 1 fold to about 1,500 fold) and H446/EP (from about 1,000 fold to about 2,500 fold). This inferred that the exogenous miR-199a-5p expression did not interaffect its endogenous expression. This independence made it a good model for studying the effect of drugs on miR-199a-5p expression because the miR-199a-5p can be fully expressed without potential negative feedback caused by the high level of miR-199a-5p.

\subsection{MiR-199a-5p regulated cisplatin-induced autophagy of H446/EP}

As we suggested that the mechanism of miR-199a-5p on drug resistance of H446 was associated with the autophagy of the cells, we determined the autophagy in the miR-199a-5p knockdown and overexpressing cells with cisplatin induction using MDC staining. Results showed that the knockdown did not affect the cisplatin-induced formation of autophagolysosomes in $\mathrm{H} 446$ but the overexpression decreased the formation of autophagolysosomes. While in H446/EP cells, where the formation of autophagolysosomes was low with cisplatin induction, the knockdown of miR-199a-5p significantly increased the formation of autophagolysosomes, but the overexpression of A549-miR-199a-5p did not affect the formation of autophagolysosomes (Fig.2D). To further confirm the role of miR-199a-5p in cisplatin-induced autophagy, we analyzed the LC3II/LC3I ratio, ATG5, and p62 expressions. Results showed the knockdown did not affect LC3II/LC3I ratio, ATG5, and p62 in H446 but the overexpression decreased LC3II/LC3I ratio, ATG5, and p62 (Fig.E1-4). While in H446/EP cells, the knockdown of miR199a-5p significantly increased LC3II/LC3I ratio, ATG5, and p62, but the overexpression of miR-199a-5p did not affect the LC3II/LC3I ratio, ATG5, and p62 (Fig.F1-4). These results were in line with the observation of the formation of autophagolysosomes. Therefore, the knockdown of miR-199a-5p did not affect cisplatin-induced autophagy in $\mathrm{H} 446$ cells, but the overexpression decreased the autophagy, while in H446/EP cells, where the cisplatin-induced autophagy was low, the knockdown of miR-199a-5p significantly increased the autophagy, but the overexpression of miR-199a-5p did not affect the autophagy. We suggested that the expression level of miR-199a-5p account for most of these results. In knockdown H446, the miR-199a-5p level was only slightly decreased thus it did not regulate cisplatininduced autophagy significantly, while in overexpressed H446, the miR-199a-5p level was high resulting in lower cisplatin-induced autophagy. On the other hand, in knockdown H446/EP, the miR-199a-5p level was significantly decreased thereby it increased cisplatin-induced autophagy, while for overexpression in $\mathrm{H} 446 / E P$, the miR-199a-5p level was already high in H446/EP that a further increase of miR-199a-5p did not further inhibit cisplatin-induced autophagy.

\subsection{MiR-199a-5p directly targeted the p62 gene.}


As our results have shown, the expression of p62 was associated with the miR-199a-5p level. Hence, we proposed that miR-199a-5p might target the p62 gene coding sequence directly. Therefore, we invested the sequence of p62 mRNA and miR-199a-5p and predicted a potential binding site with five consecutive base pairings including 4 C-G base pairs (Fig.2G1). To test this hypothesis, we conducted the DualLuciferase Reporter Assay in H446 to validate the binding of miR-199a-5p to the p62 mRNA at this predicted site. The Luciferase Reporter gene was cloned with a wild-type p62 or a p62 with mutations at the predicted site (Fig.2G2). Results showed that the overexpression of miR-199a-5p reduced the luciferase signal of samples from cells with wild-type p62 coding sequence, but it failed to affect the luciferase signal of samples from cells with mutations at the predicted site (Fig.2G3). This indicated that the miR-199a-5p only bond to wild-type p62 mRNA but not to mutated p62 mRNA. This experiment validated the direct binding of miR-199a-5p to p62 mRNA. We suggested that this binding leads to the subsequent degradation of the p62 mRNA, which is the mechanism for miR-199a-5p down-regulating p62 expression in $\mathrm{H} 446$ cells.

\section{7 $\beta$-Elemene prevented the miR-199a-5p increase induced by cisplatin}

As we have found that the miR-199a-5p was overexpressed in H446/EP cells compared with H446 cells, we were interested in how the miR-199a-5p level altered during the development of cisplatin resistance and whether $\beta$-elemene affected this process. Therefore, we exposed the cells to cisplatin and $\beta$-elemene and observed the miR-199a-5p level at different time points in H446, H446 overexpressing miR-199a-5p, H446/EP, an H446/EP miR-199a-5p knocked down. We omitted the miR-199a-5p knocked down H446 and H446/EP overexpressing miR-199a-5p because we have demonstrated that they were not much different from their parental cells in terms of responding to cisplatin and $\beta$-elemene. Results showed that, in $\mathrm{H} 446$ cells, $\beta$-elemene alone showed no significant effect on miR-199a-5p expression. Although cisplatin significantly induced the increase of miR-199a-5p after 24 and $48 \mathrm{~h}$, when it applied with $\beta$-elemene, miR199a-5p did not increase miR-199a-5p (Fig.3A). This indicated that $\beta$-elemene prevented the miR-199a-5p increase induced by cisplatin, which, we suggested, was one of the mechanisms of $\beta$-elemene preventing cisplatin resistance. Interestingly, although the H446/EP had been exposed to etoposide and cisplatin during selection, miR-199a-5p expression was further increased with cisplatin exposure (Fig.3B). A similar effect of cisplatin was also observed in H466 overexpressing miR-199a-5p and H446/EP with miR-199a$5 p$ knocked down (Fig.3CD). This inferred that the exogenous miR-199a-5p expression was independent of its endogenous expression as above mentioned. We supposed that this increase might be transient and if we subsequently cultured the cells without cisplatin, it would recover to the level of $0 \mathrm{~h}$ which was the stable level of miR-199a-5p. In addition, our results revealed that this potential transient increase of miR-199a-5p was not observed when $\beta$-elemene was applied alone or cisplatin combined with $\beta$-elemene in H446/EP cells (Fig.3D). This further confirmed that $\beta$-elemene prevented the miR-199a-5p increase induced by cisplatin.

\subsection{The combination of cisplatin and $\beta$-elemene decreased miR-199a-5p expression.}


We have demonstrated that in H446 cells with a low level of miR-199a-5p (H446 cells and H446/EP with miR-199a-5p knockdown), $\beta$-elemene prevented the miR-199a-5p increase induced by cisplatin. Then we focused on H446 cells with a high level of miR-199a-5p (H446 cells with miR-199a-5p overexpression and H446/EP) and investigated whether the combination of cisplatin and $\beta$-elemene could decrease miR$199 a-5 p$ expression. Results showed that $\beta$-elemene alone as a control did not significantly affect miR$199 a-5 p$ expression, but the combination of cisplatin and $\beta$-elemene significantly reduced the level of miR-199a-5p in both H446 cells with miR-199a-5p overexpression and H446/EP. These results inferred that miR-199a-5p was a critical target of the $\beta$-elemene and cisplatin action.

\subsection{Cisplatin and $\beta$-elemene regulated autophagy through regulating miR-199a-5p}

To obtain direct evidence that $\beta$-elemene prevents cisplatin resistance of $\mathrm{H} 446$ by targeting the autophagy regulation of miR-199a-5p, we determined the autophagy in H446 overexpressing miR-199a$5 p$ and H446/EP by analyzing LC3II/LC3I ratio, ATG5, and p62, and MDC staining. Results came out that in $\mathrm{H} 446$, the overexpression altered the response of cells to cisplatin and $\beta$-elemene. In the negative control, cisplatin alone increased LC3II/LC3I ratio, ATG5, p62, and the formation of autophagolysosomes, while with miR-199a-5p overexpressed, cisplatin increased LC3II/LC3I ratio, ATG5, p62, and the formation of autophagolysosomes only with $\beta$-elemene (Fig.3EF). On the other hand, the autophagy indicators of H446/EP cells were sensitive to cisplatin only at the presence of $\beta$-elemene, while when miR-199a-5p was knocked down, H446/EP cells became sensitive to cisplatin without $\beta$-elemene. These results suggested an essential role of miR-199a-5p in the effect of $\beta$-elemene and cisplatin and were direct evidence supporting the conclusion that $\beta$-elemene prevents cisplatin resistance of $\mathrm{H} 446$ by targeting the autophagy regulation of miR-199a-5p.

\section{Discussion}

Small cell lung cancer is a type of highly aggressive lung cancer. As it typically causes symptoms in early-stage patients, it can be discovered at earlier stages, therefore, chemotherapy drugs are usually implemented almost throughout the entire course of treatment, causing more chance for the cells to develop MDR [41]. The doses of chemotherapy are critical for the treatment of SCLC. In this study, we conducted a series of CCK-8 assays to determine the effective doses of five commonly used chemo agents including cisplatin, etoposide, paclitexal, epirubicin, and irinotecan with H446 and H446/EP. The CCK-8 assay was a simple-step cell viability assay with stable results [42] and has fewer steps than the MTT assay $[43,44]$. Thus CCK-8 was conducive for our serious viability assay. The evaluation of $I_{50}$ and DRIC revealed that $\beta$-elemene prevented $\mathrm{H} 446$ cells from the drug resistance of all five tested chemotherapeutic agents. These results partly accounted for some clinical SCLC cases that traditional medicine prescription of herbs containing $\beta$-elemene sensitized the prolonged chemotherapy [45]. Although clinical cancer treatment involves many drugs that might potentially make a difference such as anesthetics [46-49], we believed $\beta$-elemene might be one of the critical ones. 
In the present study, we selected a commonly used chemo cisplatin to further explore the actions of $\beta$ elemene which sensitized cells to cisplatin about five folds. Many previous studies have reported that cisplatin can induce MDR in H446 cells through multiple pathways [50, 51]. In this study, we used cisplatin at $5 \mu \mathrm{g} / \mathrm{ml}$ because it suppressed cell viability of $\mathrm{H} 446$ and H446/EP with $\beta$-elemene but not H446/EP without $\beta$-elemene, hence it can be used to compare cisplatin treatment with or without $\beta$ elemene. Our results demonstrated that the drug resistance to cisplatin was resulted (at least partly) from the insensitivity of autophagy induction. Accumulating literature reported that abnormal autophagy plays a critical role in cancer MDR development [52]. Nevertheless, to date, few researchers are studying the inhibition of autophagy in drug-resistant lung cancer cells. In the present study, we demonstrated that autophagy was involved in H446/EP, and the potential mechanism included the activation of LC3I/LC3II conversion, ATG5 expression, and p62 expression. LC3II conversed from LC3I has been wildly accepted to be associated with the movement of mature autophagosomes along microtubular tracks [53], while ATG5 has been one of the indicators for autophagy and it plays essential roles in the elongation and expansion of phagophore membrane. The downregulation of ATG5 could prevent the autophagosome from maturation and thereby block autophagy [54]. The p62 protein, also named SQSTM1, is involved in various signaling pathways and cellular functions including autophagy [55]. These three indicators combined with the MDC assay can be used to observed autophagy comprehensively. Our result showed that the combination of cisplatin and $\beta$-elemene can significantly increase the autophagy of multi-drug resistance sub-cell line H466/EP compared to the use of cisplatin alone.

Growing lines of evidence supported the abnormal expression of miR-199a-5p in MDR cell lines. A study showed that cisplatin-induced the decrease of miR-199a-5p expression in human osteosarcoma cells MG63 [56]. Another study reported that the expression of miR-199a-5p in leukemia cells from relapsed/refractory patients was lower than that from patients with complete remission [57]. However, our results showed that H446/EP expressed a higher level of miR-199a-5p, and cisplatin-induced an increase of miR-199a-5p expression in H446 after $24 \mathrm{~h}$. We suggested that the reaction of miR-199a-5p expression to cisplatin was cancer-type specific. Our study was the first paper that reported an abnormally high expression of miR-199a-5p in drug resistance lung cancer cells and our study is conducive to the development of miR-199a-5p as a potential biomarker for the occurrence of drug resistance in lung cancer cells. Another striking finding of this study was that miR-199a-5p could directly bind to p62 mRNA resulting in the degradation of p62 in autophagy repressive H446/EP cells. Our data suggested that miR199a-5p could be a pharmacological target for p62 protein and it was critical in mediating autophagy regulation of $\beta$-elemene and cisplatin. The role of the miR-199a-5p in autophagy regulation cisplatin and $\beta$-elemene was illustrated in Fig.4. In addition, sodium homeostasis has been suggested to be involved in autophagy, but whether the role of sodium channels in cancer [58] is associated with autophagy, $\beta$ elemene, and MDR needs further investment.

\section{Conclusions}

$\beta$-Elemene prevented multidrug resistance of small cell lung cancer cell line $\mathrm{H} 446$ and $\beta$-elemene prevents cisplatin resistance of $\mathrm{H} 446$ by targeting the autophagy regulation of miR-199a-5p. 


\section{Abbreviations}

overexpressed (OE)

knocked down (KD)

non-small cell lung cancers (NSCLC)

small cell lung cancer (SCLC)

multidrug resistance (MDR)

overexpressed negative control (OENC)

knockdown negative control (KDNC)

wild-type (WT)

monodansylcadaverine (MDC)

\section{Declarations}

\section{Availability of data and materials}

The raw data of this study are provided from the corresponding author with a reasonable request.

\section{Competing interests}

The authors claimed that there is no conflict of interest.

\section{Consent for publication}

All the authors have given their consent for this publication.

\section{Ethical approval}

Ethical approval was sought from an ethics committee of Hebei Medical University prior to commencing this study.

\section{Funding}

This study was supported by the Hebei Medical University

. The funder provided funding for the cost of the use of the equipment and the purchases of all materials.

\section{Authors' contributions}


TL contributed to the design of the study and most experimental work. HZhang, ZW, SG, XZ, NW, and HZhu contributed to the data analysis and the drafting of the manuscript. HL supervised the project. All authors had given final approval of the version to be published.

\section{Acknowledgments}

None.

\section{References}

1. Skřičková J, Kadlec B, Venclíček O, Merta Z: Lung cancer. Casopis lekaru ceskych 2018, 157(5):226-236.

2. Bade BC, Dela Cruz CS: Lung Cancer 2020: Epidemiology, Etiology, and Prevention. Clinics in chest medicine 2020, 41(1):1-24.

3. Collins LG, Haines C, Perkel R, Enck RE: Lung cancer: diagnosis and management. American family physician 2007, 75(1):56-63.

4. Saltos A, Shafique M, Chiappori A: Update on the Biology, Management, and Treatment of Small Cell Lung Cancer (SCLC). Frontiers in oncology 2020, 10:1074.

5. Zahreddine $\mathrm{H}$, Borden KL: Mechanisms and insights into drug resistance in cancer. Front Pharmacol 2013, 4:28.

6. Wu Q, Yang Z, Nie Y, Shi Y, Fan D: Multi-drug resistance in cancer chemotherapeutics: mechanisms and lab approaches. Cancer letters 2014, 347(2):159-166.

7. $\quad$ Xu Z, Jiang H, Zhu Y, Wang H, Jiang J, Chen L, Xu W, Hu T, Cho CH: Cryptotanshinone induces ROS-dependent autophagy in multidrug-resistant colon cancer cells. Chemico-biological interactions 2017, 273:48-55.

8. Zhang X, Chen X, Guo Y, Jia HR, Jiang YW, Wu FG: Endosome/lysosome-detained supramolecular nanogels as an efflux retarder and autophagy inhibitor for repeated photodynamic therapy of multidrugresistant cancer. Nanoscale horizons 2020, 5(3):481-487.

9. Utaipan T, Athipornchai A, Suksamrarn A, Chunsrivirot S, Chunglok W: Isomahanine induces endoplasmic reticulum stress and simultaneously triggers p38 MAPK-mediated apoptosis and autophagy in multidrug-resistant human oral squamous cell carcinoma cells. Oncol Rep 2017, 37(2):1243-1252.

10. Mizushima N, Levine B, Cuervo AM, Klionsky DJ: Autophagy fights disease through cellular selfdigestion. Nature 2008, 451(7182):1069-1075.

11. Feng Y, He D, Yao Z, Klionsky DJ: The machinery of macroautophagy. Cell Res 2014, 24(1):24-41. 

$\mathrm{K}$ : Autophagy and autophagic cell death are next targets for elimination of the resistance to tyrosine kinase inhibitors. Cancer Sci 2008, 99(11):2200-2208.

13. Hu YL, Jahangiri A, Delay M, Aghi MK: Tumor cell autophagy as an adaptive response mediating resistance to treatments such as antiangiogenic therapy. Cancer Res 2012, 72(17):4294-4299.

14. Chen G, Wang C, Wang J, Yin S, Gao H, Xiang LU, Liu H, Xiong Y, Wang P, Zhu X et al: Antiosteoporotic effect of icariin in ovariectomized rats is mediated via the Wnt/beta-catenin pathway. Experimental and therapeutic medicine 2016, 12(1):279-287.

15. Liu H, Xiong Y, Wang H, Yang L, Wang C, Liu X, Wu Z, Li X, Ou L, Zhang R et al: Effects of water extract from epimedium on neuropeptide signaling in an ovariectomized osteoporosis rat model. Journal of ethnopharmacology 2018, 221:126-136.

16. Liu H, Xiong Y, Zhu X, Gao H, Yin S, Wang J, Chen G, Wang C, Xiang L, Wang P et al: Icariin improves osteoporosis, inhibits the expression of PPARgamma, C/EBPalpha, FABP4 mRNA, N1ICD and jagged 1 proteins, and increases Notch 2 mRNA in ovariectomized rats. Experimental and therapeutic medicine 2017, 13(4):1360-1368.

17. Haixia W, Shu M, Li Y, Panpan W, Kehuan S, Yingquan X, Hengrui L, Xiaoguang L, Zhidi W, Ling O: Effectiveness associated with different therapies for senile osteopo-rosis: a network Meta-analysis. $J$ Tradit Chin Med 2020, 40(1):17-27.

18. Wang C, Chen G, Wang J, Liu H, Xiong Y, Wang P, Yang L, Zhu X, Zhang R: Effect of Herba Epimedium Extract on Bone Mineral Density and Microstructure in Ovariectomised Rat. Journal of Pharmaceutical and Biomedical Sciences 2016, 6(5).

19. Zhang GN, Ashby CR, Jr., Zhang YK, Chen ZS, Guo H: The reversal of antineoplastic drug resistance in cancer cells by $\beta$-elemene. Chinese journal of cancer 2015, 34(11):488-495.

20. !!! INVALID CITATION !!! \{Lee, 2018 \#1347\}.

21. Zhai B, Zhang N, Han X, Li Q, Zhang M, Chen X, Li G, Zhang R, Chen P, Wang W et al: Molecular targets of $\beta$-elemene, a herbal extract used in traditional Chinese medicine, and its potential role in cancer therapy: A review. Biomedicine \& pharmacotherapy = Biomedecine \& pharmacotherapie 2019, 114:108812.

22. Guan C, Liu W, Yue Y, Jin H, Wang X, Wang XJ: Inhibitory effect of $\beta$-elemene on human breast cancer cells. International journal of clinical and experimental pathology 2014, 7(7):3948-3956.

23. Yu X, Xu M, Li N, Li Z, Li H, Shao S, Zou K, Zou L: $\beta$-elemene inhibits tumor-promoting effect of M2 macrophages in lung cancer. Biochemical and biophysical research communications 2017, 490(2):514520. 
24. Deng M, Liu B, Song H, Yu R, Zou D, Chen Y, Ma Y, Lv F, Xu L, Zhang Z et al: $\beta$-Elemene inhibits the metastasis of multidrug-resistant gastric cancer cells through miR-1323/Cbl-b/EGFR pathway. Phytomedicine : international journal of phytotherapy and phytopharmacology 2020, 69:153184.

25. Li J, JunYu, Liu A, Wang Y: $\beta$-Elemene against human lung cancer via up-regulation of P53 protein expression to promote the release of exosome. Lung cancer (Amsterdam, Netherlands) 2014, 86(2):144150.

26. Li Y, Jiang W, Hu Y, Da Z, Zeng C, Tu M, Deng Z, Xiao W: MicroRNA-199a-5p inhibits cisplatininduced drug resistance via inhibition of autophagy in osteosarcoma cells. Oncol Lett 2016, 12(5):42034208.

27. Ahmadi A, Khansarinejad B, Hosseinkhani S, Ghanei M, Mowla SJ: miR-199a-5p and miR-495 target GRP78 within UPR pathway of lung cancer. Gene 2017, 620:15-22.

28. Hua Q, Jin M, Mi B, Xu F, Li T, Zhao L, Liu J, Huang G: LINC01123, a c-Myc-activated long noncoding RNA, promotes proliferation and aerobic glycolysis of non-small cell lung cancer through miR199a-5p/c-Myc axis. Journal of hematology \& oncology 2019, 12(1):91.

29. Li Y, Wang D, Li X, Shao Y, He Y, Yu H, Ma Z: MiR-199a-5p suppresses non-small cell lung cancer via targeting MAP3K11. Journal of Cancer 2019, 10(11):2472-2479.

30. Li DJ, Wang X, Yin WH, Niu K, Zhu W, Fang N: MiR-199a-5p suppresses proliferation and invasion of human laryngeal cancer cells. European review for medical and pharmacological sciences 2020, 24(23):12200-12207.

31. Z Zhu QD, Zhou QQ, Dong L, Huang Z, Wu F, Deng X: MiR-199a-5p Inhibits the Growth and Metastasis of Colorectal Cancer Cells by Targeting ROCK1. Technology in cancer research \& treatment 2018, 17:1533034618775509.

32. Li Y, Zhang G, Wu B, Yang W, Liu Z: miR-199a-5p Represses Protective Autophagy and Overcomes Chemoresistance by Directly Targeting DRAM1 in Acute Myeloid Leukemia. J Oncol 2019, 2019:56134175613434.

33. Chen PH, Liu AJ, Ho KH, Chiu YT, Anne Lin ZH, Lee YT, Shih CM, Chen KC: microRNA-199a/b-5p enhance imatinib efficacy via repressing WNT2 signaling-mediated protective autophagy in imatinibresistant chronic myeloid leukemia cells. Chem Biol Interact 2018, 291:144-151.

34. Pan B, Chen Y, Song H, Xu Y, Wang R, Chen L: Mir-24-3p downregulation contributes to VP16-DDP resistance in small-cell lung cancer by targeting ATG4A. Oncotarget 2015, 6(1):317-331.

35. Wu Z, Ou L, Wang C, Yang L, Wang P, Liu H, Xiong Y, Sun K, Zhang R, Zhu X: Icaritin induces MC3T3-E1 subclone14 cell differentiation through estrogen receptor-mediated ERK1/2 and p38 signaling activation. Biomedicine \& pharmacotherapy = Biomedecine \& pharmacotherapie 2017, 94:1-9. 
36. Liu X, Liu H, Xiong Y, Yang L, Wang C, Zhang R, Zhu X: Postmenopausal osteoporosis is associated with the regulation of SP, CGRP, VIP, and NPY. Biomedicine \& pharmacotherapy = Biomedecine \& pharmacotherapie 2018, 104:742-750.

37. Xue JF, Shi ZM, Zou J, Li XL: Inhibition of PI3K/AKT/mTOR signaling pathway promotes autophagy of articular chondrocytes and attenuates inflammatory response in rats with osteoarthritis. Biomedicine \& pharmacotherapy = Biomedecine \& pharmacotherapie 2017, 89:1252-1261.

38. Li X, Peng B, Zhu X, Wang P, Xiong Y, Liu H, Sun K, Wang H, Ou L, Wu Z et al: Changes in related circular RNAs following ERbeta knockdown and the relationship to rBMSC osteogenesis. Biochemical and biophysical research communications 2017, 493(1):100-107.

39. Clément T, Salone V, Rederstorff M: Dual luciferase gene reporter assays to study miRNA function. Methods in molecular biology (Clifton, NJ) 2015, 1296:187-198.

40. Li R, Xiao C, Liu H, Huang Y, Dilger JP, Lin J: Effects of local anesthetics on breast cancer cell viability and migration. $B M C$ cancer 2018, 18(1):666.

41. Byers LA, Rudin CM: Small cell lung cancer: where do we go from here? Cancer 2015, 121(5):664672.

42. Liu H, Dilger JP, Lin J: Effects of local anesthetics on cancer cells. Pharmacology \& Therapeutics 2020, 212:107558.

43. Liu H, Dilger JP, Lin J: Lidocaine Suppresses Viability and Migration of Human Breast Cancer Cells: TRPM7 as A Target for Some Breast Cancer Cell Lines. Cancers 2021, 13(2):234.

44. Liu H, Dilger JP, Lin J: The Role of Transient Receptor Potential Melastatin 7 (TRPM7) in Cell Viability: A Potential Target to Suppress Breast Cancer Cell Cycle. Cancers 2020, 12(1).

45. Cao M, Long M, Chen Q, Lu Y, Luo Q, Zhao Y, Lu A, Ge C, Zhu L, Chen Z: Development of $\beta$-elemene and Cisplatin Co-Loaded Liposomes for Effective Lung Cancer Therapy and Evaluation in Patient-Derived Tumor Xenografts. Pharmaceutical research 2019, 36(8):121.

46. Li R, Liu H, Dilger JP, Lin J: Effect of Propofol on breast Cancer cell, the immune system, and patient outcome. BMC anesthesiology 2018, 18(1):77.

47. Li R, Huang Y, Liu H, Dilger JP, Lin J: Comparing volatile and intravenous anesthetics in a mouse model of breast cancer metastasis. In., vol. 78: American Association for Cancer Research; 2018: 2162.

48. Liu H: A Prospective for the Potential Effect of Local Anesthetics on Stem-Like Cells in Colon Cancer. Biomedical Journal of Scientific \& Technical Research 2020, 25(2):18927-18930. 
49. Liu H: A clinical mini-review: Clinical use of Local anesthetics in cancer surgeries. The Gazette of Medical Sciences 2020, 1(3):030-034.

50. Li W, Shi Y, Wang R, Pan L, Ma L, Jin F: Resveratrol promotes the sensitivity of small-cell lung cancer H446 cells to cisplatin by regulating intrinsic apoptosis. Int J Oncol 2018, 53(5):2123-2130.

51. Liu HN, Qie P, Yang G, Song YB: miR-181b inhibits chemoresistance in cisplatin-resistant $\mathrm{H} 446$ small cell lung cancer cells by targeting Bcl-2. Archives of medical science : AMS 2018, 14(4):745-751.

52. Li YJ, Lei YH, Yao N, Wang CR, Hu N, Ye WC, Zhang DM, Chen ZS: Autophagy and multidrug resistance in cancer. Chinese journal of cancer 2017, 36(1):52.

53. Xie R, Nguyen S, McKeehan WL, Liu L: Acetylated microtubules are required for fusion of autophagosomes with lysosomes. BMC cell biology 2010, 11:89.

54. Arakawa S, Honda S, Yamaguchi H, Shimizu S: Molecular mechanisms and physiological roles of Atg5/Atg7-independent alternative autophagy. Proceedings of the Japan Academy Series B, Physical and biological sciences 2017, 93(6):378-385.

55. Tao M, Liu T, You Q, Jiang Z: p62 as a therapeutic target for tumor. European journal of medicinal chemistry 2020, 193:112231.

56. Li Y, Jiang W, Hu Y, Da Z, Zeng C, Tu M, Deng Z, Xiao W: MicroRNA-199a-5p inhibits cisplatininduced drug resistance via inhibition of autophagy in osteosarcoma cells. Oncology letters 2016, 12(5):4203-4208.

57. Li Y, Zhang G, Wu B, Yang W, Liu Z: miR-199a-5p Represses Protective Autophagy and Overcomes Chemoresistance by Directly Targeting DRAM1 in Acute Myeloid Leukemia. J Oncol 2019, 2019:56134175613417.

58. Liu H: Nav channels in cancers: Nonclassical roles. Global Journal of Cancer Therapy 2020, 6(1):5.

\section{Table 1}

Table 1. DRIC of multidrug in $\mathrm{H} 446$. 


\begin{tabular}{|c|c|c|c|c|c|c|c|}
\hline & \multicolumn{3}{|c|}{$0 \mu \mathrm{g} / \mathrm{ml} \beta$-Elemene } & \multicolumn{4}{|c|}{$5 \mu \mathrm{g} / \mathrm{ml} \beta$-Elemene } \\
\hline & \multicolumn{3}{|c|}{$\mathrm{IC}_{50}($ mean $\pm S D)$} & \multicolumn{4}{|c|}{$\mathrm{IC}_{50}($ mean $\pm S D)$} \\
\hline $\begin{array}{l}\text { Drug } \\
(\mu \mathrm{g} / \mathrm{ml})\end{array}$ & $\mathrm{H} 446$ & H446/EP & DRIC & $\mathrm{H} 446$ & H446/EP & DRIC & $\begin{array}{l}\text { DRIC } \\
\text { FC }\end{array}$ \\
\hline cisplatin & $0.59 \pm 0.33$ & $17.67 \pm 2.01^{\mathrm{a}}$ & 29.95 & $0.57 \pm 0.22$ & $3.17 \pm 1.61^{b}$ & 5.56 & 0.19 \\
\hline etoposide & $0.38 \pm 0.19$ & $22.21 \pm 2.12^{\mathrm{a}}$ & 58.45 & $0.45 \pm 0.23$ & $12.56 \pm 1.67^{b}$ & 27.9 & 0.47 \\
\hline paclitexal & $0.14 \pm 0.09$ & $0.78 \pm 0.89^{a}$ & 5.57 & $0.21 \pm 0.15$ & $0.31 \pm 0.89^{b c}$ & 1.48 & 0.27 \\
\hline epirubicin & $0.61 \pm 0.22$ & $5.98 \pm 0.89^{a}$ & 9.80 & $0.69 \pm 0.32$ & $1.11 \pm 0.59^{b c}$ & 3.06 & 0.31 \\
\hline irinotecan & $18.98 \pm 2.11$ & $110.86 \pm 4.89^{a}$ & 5.84 & $15.32 \pm 2.02 a$ & $69.98 \pm 4.34^{b}$ & 1.61 & 0.27 \\
\hline vinorelbine & $4.45 \pm 0.98$ & $9.32 \pm 1.99^{a}$ & 2.09 & $4.95 \pm 1.22 \mathrm{a}$ & $5.19 \pm 1.15^{b}$ & 1.05 & 0.50 \\
\hline
\end{tabular}

Note: "a" compared to $0 \mu \mathrm{g} / \mathrm{ml}$-Elemene H446, "b" compared to $0 \mu \mathrm{g} / \mathrm{ml} \beta$-Elemene H446/EP, "c" compared to $5 \mu \mathrm{g} / \mathrm{ml} \beta$-Elemene H446. SD, standard deviation; DRIC, drug resistance indices for cancer; FC, fold change.

\section{Figures 1-4}

Figures 1-4 are not available with this version.

\section{Figure legend:}

Figure 1. $\beta$-Elemene decreased DRIC of multiple chemotherapeutic agents in $\mathrm{H} 446$. A. Effect of $\beta$-elemene on cell viability of H446, H446/EP, and HULEC-5a. The cell viability was measured using CCK-8 $24 \mathrm{~h}$ after exposure to the drugs. B-C. LC3II/LC3I, ATG5, and p62 expressions in H446 and H446/EP after drug exposure. The expression was measured using the western blotting assay. E. Representative images of BC. F. Image of autophagolysosomes in cells with drug exposure. The fluorescence images of autophagolysosomes were captured after the MDC staining of cells (magnification $\times 200) .\left({ }^{*} p<0.01\right)$

Figure 2. MiR-199a-5p regulated cisplatin-induced autophagy of H446/EP by inhibiting p62 and ATG5. A. MiR-199a-5p expression in H446 and H446/EP. MiR-199a-5p expression was determined using the QPCR assay. B. MiR-199a-5p expression in H446 with miR-199a-5p knockdown or overexpression. (OENC: overexpression negative control; OE: MiR-199a-5p overexpression; KDNC: knockdown negative control; KD: MiR-199a-5p knockdown) C. MiR-199a-5p expression in H446/EP with miR-199a-5p knockdown or overexpression. D. Image of autophagolysosomes in miR-199a-5p knocked down or overexpressed cells with cisplatin exposure. The autophagolysosomes were observed using an MDC assay (magnification 
x200). E1-4. LC3II/LC3I, ATG5, and p62 expressions in miR-199a-5p knocked down or overexpressed H446. The protein expression was determined using the western blotting assay. F1-4 LC3II/LC3I, ATG5, and p62 expressions in miR-199a-5p knocked down or overexpressed H446/EP. G1. The predicted binding site of miR-199a-5p to p62 mRNA. G2. Luciferase reporter gene sequence with the alignment of the miR199a-5p gene at the predicted binding site in Dual-Luciferase Reporter Assay. G3. Effects of miR-199a-5p on luciferase signal in H446. H446 cells were co-transfected with miR-199a-5p expression vectors and wild-type (WT)-p62 or mutated-p62 vectors. The luciferase signal was determined $24 \mathrm{~h}$ after the transfection followed by the addition of substrate. $\left({ }^{\star} p<0.01\right)$

Figure 3. $\beta$-Elemene regulated the effect of cisplatin on MiR-199a-5p. A. The effect of cisplatin and $\beta$ elemene on miR-199a-5p expression in H446. MiR-199a-5p expression was determined using the QPCR assay. B. The effect of cisplatin and $\beta$-elemene on miR-199a-5p expression in H446/EP. C. The effect of cisplatin and $\beta$-elemene on miR-199a-5p expression in miR-199a-5p knocked down H446/EP. D. The effect of cisplatin and $\beta$-elemene on miR-199a-5p expression in miR-199a-5p overexpressed H446. (OENC: overexpression negative control; OE: MiR-199a-5p overexpression; KDNC: knockdown negative control; KD: MiR-199a-5p knockdown) E1-4. LC3II/LC3I, ATG5, and p62 expressions in cells. The protein expression was determined using the western blotting assay. F. Image of autophagolysosomes in miR199a-5p knocked down H446/EP or overexpressed H446 with cisplatin and $\beta$-elemene exposure. The autophagolysosomes were observed using the MDC assay (magnification $\times 200) .\left({ }^{*} p<0.01\right)$

Figure 4. $\beta$-Elemene prevents cisplatin resistance of $\mathrm{H} 446$ by targeting the autophagy regulation of miR199a-5p. 